\title{
Modelamiento de nichos ecológicos de flora amenazada para escenarios de cambio climático en el departamento de Tacna - Perú
}

\section{Modeling ecological niches of threatened flora for climate change scenarios in Tacna department - Peru}

\author{
Marco Alberto Navarro Guzmán ${ }^{10}$, Cesar Augusto Jove Chipana² ${ }^{[0}$ \\ y Javier Máximo Ignacio Apaza ${ }^{3}$
}

Navarro-Guzmán, M.A., Jove-Chipana, C.A. y Apaza, J.M. (2020). Modelamiento de nichos ecológicos de flora amenazada para escenarios de cambio climático en el departamento de Tacna - Perú. Colombia forestal, 23(1), $51-67$.

Recepción: 7 de mayo 2019

\section{Resumen}

A pesar de la numerosa información científica sobre cambio climático mundial, no existen estudios que demuestren los efectos sobre la biodiversidad a menor escala. Por ello, utilizando 19 variables bioclimáticas, cinco de radiación solar, altitud, software especializado (MaxEnt) y coordenadas geográficas de presencia de cinco especies de flora categorizada verificadas en campo se modelaron sus nichos ecológicos actuales y proyectados a los cuatro escenarios futuros de emisiones (2050 y 2070). Se demostró que el de Buddleja coriacea disminuirá en más del $80 \%$ por las variaciones futuras de precipitación y temperatura consecuencia del cambio climático, mientras que Carica candicans, Haplorhus peruviana, Kageneckia lanceolata y Weberbauerella brongniartioides se mantendrán e incrementarán, deduciéndose que sus categorías de amenaza derivan principalmente por actividad antropogénica. Estos modelos mejoran significativamente la comprensión del funcionamiento ecosistémico, otorgando información útil para diseñar políticas y acciones de
Aprobación: 16 de diciembre 2019

conservación que orienten la gestión territorial hacia la estrategia de adaptación basada en ecosistemas.

Palabras clave: GEI, MaxEnt, Peligro Crítico, RCP, Tacna, Worldclim.

\begin{abstract}
Despite the numerous scientific information on global climate change, there are no studies that show effects on biodiversity on a smaller scale. Therefore, using 19 bioclimatic variables, five solar radiation, altitude, specialized software (MaxEnt) and geographical coordinates of the presence of five species of categorized flora verified in the field, their current ecological niches were modeled and projected to the four future emission scenarios (2050 and 2070) showing that Buddleja coriacea will decrease by more than $80 \%$ due to future variations in precipitation and temperature due to climate change, while Carica candicans, Haplorhus peruviana, Kageneckia lanceolata and Weberbauerella brongniartioides will remain and increase, deducing that Its threat categories derive mainly from anthropogenic activity.
\end{abstract}

1 Facultad de Ingeniería Ambiental de la Universidad Latinoamericana CIMA de Tacna. Tacna, Perú. mnavarro@cima.edu.pe. Autor para correspondencia.

2 Herbario Takana, Universidad Nacional Jorge Basadre Grohmann de Tacna. Tacna, Perú. cesarjove@gmail.com

3 Herbario Takana, Universidad Nacional Jorge Basadre Grohmann de Tacna. Tacna, Perú. jmignacionet@gmail.com 
These models significantly improve the understanding of ecosystem functioning, providing useful information to design conservation policies and actions that guide territorial management towards the Ecosystem-based Adaptation strategy.

Keywords: GEI, MaxEnt, Critically Endangered, RCP, Tacna, Worldclim.

\section{INTRODUCCIÓN}

Según el Minam (2015), los impactos del cambio climático son hechos innegables y evidentes que afectan a los ecosistemas, diversidad biológica, la base de recursos naturales, su distribución geográfica, la estructura productiva y de servicios, así como la infraestructura económica y social, la salud y bienestar de la población, familias e individuos. En zonas altoandinas los efectos a la biodiversidad son aún mayores, ya que según Castaño (2002) la fragilidad del espacio reducido y específico se vuelve un agravante para la flora y la fauna. Kappelle y Horn (2005) indican que cuando las condiciones no permiten la migración altitudinal de las especies, el ecosistema es igual de vulnerable que una isla pequeña incrementando el riesgo a la extinción.

Se considera que una especie de flora se encuentra amenazada cuando sus poblaciones silvestres se reducen extremadamente por el desarrollo de actividades humanas o por efecto del cambio climático, y son protegidas estatalmente con base en las clasificaciones en el que se incluyen criterios de la UICN (2012) como son: peligro crítico (CR), en peligro (EN), vulnerable (VU) y casi amenazado (NT) como la lista oficial de especies de flora categorizada del Perú (Decreto Supremo N. ${ }^{\circ 043-2006-A G, ~ 2006) . ~ A d e m a ́ s, ~ e x i s t e ~ e l ~ L i s t a-~}$ do de especies de flora silvestre Cites que tiene la finalidad de velar por que el comercio internacional de especímenes silvestres no constituya una amenaza para su supervivencia, en el cual se ofrecen diversos grados de protección a más de 30000 especies en el ámbito mundial (Minam, 2018).

Este grado de amenaza puede incrementarse debido al aumento de emisiones de GEI que el
IPCC (2013) las define como las cuatro trayectorias de concentración representativas (RCP por sus siglas en inglés) en función de la concentración de $\mathrm{CO}_{2}$ en la atmosfera (forzamiento radiactivo total para el 2100 que oscilará entre 2.6 y 8.5 W.m ${ }^{-2}$ y será mayor que en la actualidad). Las RCP comprenden un escenario en el que los esfuerzos en mitigación conducen a un nivel de forzamiento muy bajo (RCP2.6), dos escenarios de estabilización (RCP4.5 y RCP6.0) y un escenario con un nivel muy alto de emisiones de GEI (RCP8.5). Las proyecciones de temperatura y precipitación en estos RCP han sido publicadas en el Worldclim (2016) útiles para el mapeo y modelado espacial.

Entonces, siendo el departamento de Tacna de poca superficie territorial, en el cual se presentan variaciones de temperaturas en diversas zonas, $\mathrm{p}$. ej. La Yarada, (Senamhi, 2010) con variaciones extremas máximas y mínimas de entre $1{ }^{\circ} \mathrm{C}$ y $1.3{ }^{\circ} \mathrm{C}$, es que se le ha considerado como uno de los primeros departamentos en donde ya se evidencian los efectos del cambio climático. Por lo anterior, el gobierno regional de Tacna (Gore Tacna), elaboró su Estrategia regional de cambio climático (ERCC) aprobada mediante Ordenanza Regional N. ${ }^{\circ} 005$ 2015-CR/GOB. REG. TACNA (2015).

En este contexto es válido preguntarse ¿cuál será la distribución geográfica actual de los nichos ecológicos de las especies de flora silvestre categorizada CR del departamento de Tacna y como cambiarán frente a las variaciones climáticas en las cuatro RCP del año 2050 y 2070?

Como respuesta se realizó el modelamiento actual y proyectado de los nichos ecológicos de las especies categorizadas CR utilizando el software MaxEnt, los datos de ocurrencia de campo y del estudio Zonificación ecológica y económica 
(ZEE) Tacna (Ordenanza Regional N. ${ }^{\circ}$ 016-2012CR/GOB. REG. TACNA, 2013) y un conjunto de variables ambientales (bioclimáticas, altitud, radiación solar, etc.) actuales y proyectadas a los RCP futuros. La información científica obtenida servirá para que se diseñen, a nivel departamental, adecuados planes de gestión territorial con eficientes medidas de adaptación y mitigación al cambio climático, que estén estrechamente vinculadas con la conservación.

\section{MATERIALES Y MÉTODOS}

\section{Área de estudio}

El departamento de Tacna está localizado en el extremo sur del Perú, limita por el noroeste con Moquegua, por el noreste con Puno, por el sur con la República de Chile, por el este con la República de Bolivia y por el oeste con el océano Pacífico (figura 1). Tiene una superficie territorial de 16

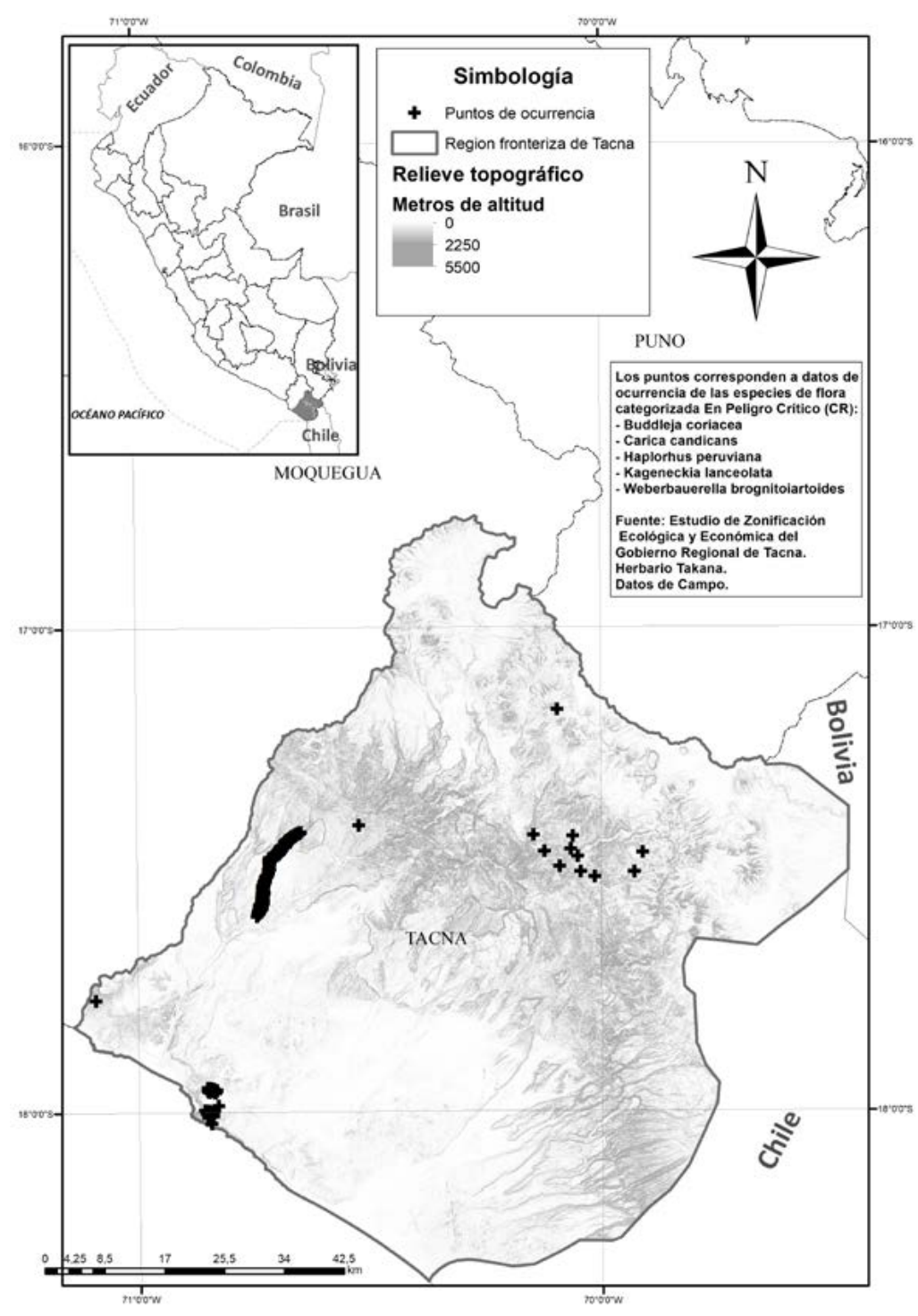

Figura 1. Mapa de localización estratégica del departamento de Tacna en Sudamérica y de distribución de las coordenadas geográficas en las que fueron reportadas las especies de flora categorizada (CR) evaluadas al interior del área de estudio. 
$075.73 \mathrm{~km}^{2}$ que representa el $1.25 \%$ del país y está dividida en cuatro provincias: Tacna, Tarata, Candarave y Jorge Basadre (Gobierno Regional de Tacna, 2014).

\section{Especies de flora silvestre categorizada evaluadas}

De acuerdo con la lista oficial de especies de flora categorizada del Perú (Decreto Supremo N. ${ }^{\circ} 043$ 2006-AG, 2006), en el departamento de Tacna se encuentran dentro de la categoría en peligro crítico (CR) a Buddleja coriacea J. Remy, Carica candicans A. Gray, Haplorhus peruviana Engl, Kageneckia lanceolata Ruiz \& Pav y Weberbauerella brongniartioides Ulbr. La cantidad de ocurrencias o coordenadas de ubicación geográfica para cada especie categorizada CR que se reportaron en la ZEE Tacna se presenta en la tabla 1, y estas fueron contrastadas con información técnica no publicada del Herbario Takana, informes técnicos de estudios poblacionales de biodiversidad realizados por el Gore Tacna, así como el estudio poblacional de $C$. candicans realizado por Franco (2013). Del mismo modo, se realizaron 10 salidas de campo con 60 puntos de búsqueda, observación y fotografiado de las especies (sin colecta) para confirmar su presencia/ausencia en las coordenadas referidas, las cuales se presentan en la figura 1. Respecto a la importancia de las especies categorizadas evaluadas, B. coriacea y $K$. lanceolata son especies forestales proveedoras de madera de excelente calidad, utilizadas en construcción de vigas, puertas, ventanas, dinteles y herramientas agrícolas (yuntas, arados, tacllas) así como artesanías y utilería; igualmente, es común verlo en compuertas y partes de los canales de regadío, además su leña y carbón son de excelente calidad (Reynel y León, 1990; Reynel y Marcelo, 2009). Asimismo, C. candicans es una planta frondosa y foliosa y juega un papel importante como captadores naturales de niebla, son productores primarios en conjunto con el estrato arbóreo arbustivo y sirven como hábitat o nicho ecológico de organismos que cumplen similares funciones dentro del ecosistema (Mendoza, 2006; Sagástegui, Rodríguez y Arroyo, 2007; Weberbauer 1945). Respecto a H. peruviana, Pumasupa (2017) determinó un stock de carbono de 4.5244 tC. ha ${ }^{-1}$, demostrando así su importancia como proveedores de servicios ecosistémicos de regulación, mientras que $W$. brongniartioides es endémica para las costas del sur del Perú del que aún no se tienen muchos datos de utilidad de la especie.

\section{Variables ambientales utilizadas}

Se utilizaron 19 variables bioclimáticas actuales, las cuales contienen información de la combinación de datos de temperatura y precipitación en

Tabla 1. Nombre científico, nombre común, usos e importancia y número de ocurrencias o coordenadas geográficas de reporte de cada una de las cinco especies de flora categorizada (CR) evaluadas en el modelamiento de nicho ecológico actual y proyectado

\begin{tabular}{|c|c|c|c|}
\hline Nombre científico & Nombre común & Importancia & Ocurrencias \\
\hline Buddleja coriacea & Kolle, Quishuar & Ornamental, madera y leña. & 1 \\
\hline Carica candicans & Papaya silvestre, mito & $\begin{array}{l}\text { Su fruto se utiliza para consumo humano, atrapa } \\
\text { niebla. }\end{array}$ & 122 \\
\hline Haplorhus peruviana & Carzo & $\begin{array}{l}\text { Servicios ambientales (fijación de } \mathrm{CO}_{2} \text {, regulación } \\
\text { de suelo y climas) }\end{array}$ & 1814 \\
\hline Kageneckia lanceolata & Lloque & $\begin{array}{l}\text { Se utiliza su madera para la construcción de herra- } \\
\text { mientas. }\end{array}$ & 11 \\
\hline Weberbauerella brognitoartioides & No tiene & Leguminosa con ausencia de estudios ecológicos. & 1 \\
\hline
\end{tabular}


diferentes épocas del año. Las mismas variables del año 2050 y 2070 corresponden a proyecciones a mediano y largo plazo de sus respectivos RCP (Hijmans et al., 2005). Bio_1 es la temperatura media anual $\left({ }^{\circ} \mathrm{C}\right)$; Bio_2 es el rango medio diurno; Bio_3 es la Isotermalidad; Bio_4 es la estacionalidad de la temperatura; Bio_5 es la temperatura máxima del mes más cálido $\left({ }^{\circ} \mathrm{C}\right)$; Bio_6 es la temperatura mínima del mes más frío $\left({ }^{\circ} \mathrm{C}\right)$; Bio_7 es el rango anual de temperatura; Bio_8 es la temperatura media del cuarto más húmedo $\left({ }^{\circ} \mathrm{C}\right)$; Bio_9 es la temperatura media del cuarto más seco $\left({ }^{\circ} \mathrm{C}\right)$; Bio_10 es la temperatura media del cuarto más caluroso $\left({ }^{\circ} \mathrm{C}\right)$; Bio_11 es la temperatura media del cuarto más frío $\left({ }^{\circ} \mathrm{C}\right)$; Bio_12 es la precipitación anual (mm); Bio_13 es la precipitación del mes más húmedo (mm); Bio_14 es la precipitación del mes más seco (mm); Bio_15 es la estacionalidad en las precipitaciones; Bio_16 es la precipitación del cuarto más húmedo $(\mathrm{mm}) ;$ Bio_17 la precipitación del cuarto más seco (mm); Bio_18 es la precipitación del cuarto más caluroso (mm); y Bio_19 es la precipitación del cuarto más frío $(\mathrm{mm})$. Además, se utilizó la variable ELE, que es la topografía terrestre representada en un raster de la estructura tridimensional de la superficie de la Tierra (Nasa, 2019).

También se utilizaron cinco variables vinculadas a la radiación solar, GHI, que es la irradiación horizontal global, DNI que es la irradiación normal directa, el DIF que representa la irradiación horizontal difusa, el GTI que indica la irradiación global inclinada y TEMP que es la temperatura del aire a dos metros del suelo, obtenidas del atlas $\mathrm{SO}^{-}$ lar resource data obtained from the Global Solar Atlas, owned by the World Bank Group and provided by Solargis (World Bank Group, 2019).

Las 25 variables fueron descargadas en formato raster de $1 \mathrm{~km}^{2}$ por píxel (Geotiff) para ejecutarles procesos previos al análisis con el software QGIS, como el recorte de cada variable a los límites del área de estudio, la transformación al formato ASCI verificando el número y tamaño de las celdas, filas y columnas, así como la proyección al sistema de coordenadas UTM WGS 84 Zona 19S para los cálculos geométricos en km² (QGIS Development Team, 2019).

\section{Análisis de datos}

El software utilizado para el modelamiento de nichos ecológicos fue el MaxEnt v3.4.1, el cual funciona con algoritmos predictores de la distribución potencial de las especies de acuerdo con las condiciones ambientales (Phillips, Anderson y Shapire, 2006). Los resultados de salida fueron en formato logistic por su facilidad de interpretación en programas de información geográfica (Phillips y Dudík, 2008).

\section{Evaluación de modelos}

Se utilizó el Area Under the Curve (AUC), estadístico que representa el área total bajo la curva roja del Receiver Operating Characteristic (ROC) y que valida los modelos elaborados en MaxEnt. Los valores de AUC de 0.5 a 0.7 tienen baja confianza, de 0.7-0.9 tienen aplicación útil en el modelo y los mayores a 0.9 tienen alta confianza (Lobo, Jiménez y Real, 2007). Para comprobar el aporte de cada variable de forma individual se utilizó la prueba de Jackknife, el cual tiene la función principal de correr el modelo con cada variable y medir su aporte (Shcheglovitova y Anderson, 2013).

\section{RESULTADOS}

Todos los modelos de nicho ecológico obtenidos para las cinco especies de flora categorizada CR elaborados en MaxEnt dieron como resultado valores estadísticos AUC mayores a 0.9, siendo el menor valor 0.905 y el mayor 0.999 , estos se consideran modelos predictivos de alta confianza.

Se obtuvieron nueve modelos de nicho ecológico para cada especie (el modelo actual más cuatro proyectados al año 2050 y para el 2070), conformando así un total 45 archivos raster del tipo ASCI que contenían la distribución geográfica de 
los nichos ecológicos de las cinco especies, tanto para la actualidad como para los cuatro RCP proyectados. En la tabla 2 se presentan los resultados de cobertura actual del nicho ecológico y el porcentaje de pérdida o ganancia de cobertura en el RCP proyectado. Allí se observa que solo la especie $B$. coriacea tendrá pérdidas de superficie de nicho ecológico en todos los RCP proyectados al 2050 y 2070 ; mientras que C. candicans y $W$. brongniartioides presentan ganancia de superficie en todos los escenarios evaluados. H. peruviana demuestra pérdida de superficie de su nicho ecológico en los tres primeros escenarios del 2050, pero a partir del RCP8.5 estos tienden a volverse positivos (ganancia), mientras que $K$. lanceolata también presenta ganancia en todas las proyecciones RCP. En la misma tabla 2 se presentan los resultados de la prueba de Jackknife, resaltándose a aquella variable que aporto mayor información al modelamiento de los nichos ecológicos por especie. Se evidencia que predominan las variables bioclimáticas, en especial las de precipitación.

\section{DISCUSIÓN}

Los resultados de AUC obtenidos durante el modelamiento de nicho ecológico de las cinco especies categorizadas CR del departamento de Tacna (mayores a 0.9) demuestran alta confiabilidad (Phillips et al., 2006) como los resultados de Reynoso et al. (2018) de 0.882 para Pinus oocarpa y 0.947 para Pinus pseudostrobus; o como los de Wan et al. (2015) entre 0.7 y 0.9 para Pinus koraiensis y Eleutherococcus senticosus; o los de Ibarra et al. (2016) para Coryphantha chihuahuensis de 0.9. Todo esto

Tabla 2. Resultados obtenidos en la predicción de los nichos ecológico para las especies de flora categorizada (CR). La fila $\mathrm{km}^{2}$ representa la superficie actual de cada especie. Las filas \% representan la ganancia o pérdida (según signo) de superficie de los nichos ecológicos de las especies en los RCP del año 2050 y 2070. Las filas Var indica el nombre de las variables que contribuyeron con mayor información en el diseño del modelo de nicho ecológico, según la prueba de Jackknife realizado con el MaxEnt

\begin{tabular}{|c|c|c|c|c|c|c|c|}
\hline \multirow{2}{*}{ Año } & \multirow{2}{*}{$\mathbf{R C P}$} & \multirow{2}{*}{ Valor } & \multicolumn{5}{|c|}{ Especies de flora categorizada CR evaluadas } \\
\hline & & & B. coriacea & C. candicans & H. peruviana & K. lanceolata & W. brongniartioides \\
\hline \multirow{2}{*}{\multicolumn{2}{|c|}{ Actual }} & $\mathrm{km}^{2}$ & 540.98 & 75.47 & 254.05 & 1135.55 & 185.94 \\
\hline & & Var & Bio_1 & Bio_12 & Bio_12 & Bio_13 & $\mathrm{DNI}$ \\
\hline \multirow{2}{*}{\multicolumn{2}{|c|}{2.6}} & $\%$ & -77.69 & 34.37 & -5.96 & 31.92 & 473.79 \\
\hline & & Var & Bio_14 & Bio_12 & Bio_7 & Bio_16 & Bio_18 \\
\hline \multirow{6}{*}{2050} & \multirow{2}{*}{4.5} & $\%$ & -58.35 & 22.72 & -13.62 & 35.69 & 497.50 \\
\hline & & Var & Bio_14 & Bio_12 & Bio_7 & Bio_13 & Bio_18 \\
\hline & \multirow{2}{*}{6.0} & $\%$ & -86.19 & 24.44 & -9.94 & 29.83 & 421.59 \\
\hline & & Var & Bio_14 & Bio_12 & Bio_7 & Bio_18 & Bio_18 \\
\hline & \multirow{2}{*}{8.5} & $\%$ & -58.97 & 39.59 & 12.33 & 16.73 & 499 \\
\hline & & Var & Bio_14 & Bio_12 & Bio_7 & Bio_18 & Bio_18 \\
\hline \multirow{8}{*}{2070} & \multirow{2}{*}{2.6} & $\%$ & -79.42 & 33.34 & 7.04 & 12.92 & 436.56 \\
\hline & & Var & Bio_14 & Bio_12 & Bio_7 & Bio_16 & Bio_18 \\
\hline & \multirow{2}{*}{4.5} & $\%$ & -59.50 & 29.93 & 9.15 & 28.09 & 373.64 \\
\hline & & Var & Bio_14 & Bio_12 & Bio_7 & Bio_18 & Bio_18 \\
\hline & \multirow{2}{*}{6.0} & $\%$ & -59.50 & 29.93 & 9.15 & 28.09 & 373.64 \\
\hline & & Var & Bio_14 & Bio_12 & Bio_7 & Bio_18 & Bio_18 \\
\hline & \multirow{2}{*}{8.5} & $\%$ & -68.54 & 38.23 & 3.42 & 22.72 & 261.31 \\
\hline & & Var & Bio_1 & Bio_12 & Bio_7 & Bio_18 & Bio_18 \\
\hline
\end{tabular}


comprueba que las variables ambientales y el software MaxEnt son las principales herramientas para predicciones de nicho ecológico (Elith et al., 2006) demostrado por Ortega y Townsend (2008) al compararlo entre seis diferentes métodos (algoritmos) resultando predicciones más significativas, sin fallas graves y con el análisis de participación de cada variable.

En general, el modelamiento de nichos ecológicos viene siendo aplicado a diferentes especies silvestres (Morales, 2012), ya sea para predecir su distribución o para gestionar oportunidades de aprovechamiento; como la publicación de Osinfor (2013) que presenta modelos de nicho ecológico para 29 especies forestales utilizando el software MaxEnt. También ha sido utilizado para determinar zonas potenciales para especies cultivables de flora con elevado valor comercial, como la VaniIla planifolia (Hernández et al., 2016) a manera de oportunidad de econegocio. Sin embargo, es necesario considerar el cuestionamiento a la utilidad de los análisis ROC al ponderar igual los errores de omisión que los de comisión (Lobo et al., 2007; Peterson, Papes y Soberón, 2008) por lo que Martínez et al., (2016) utilizaron el programa Tool for Partial-ROC (Narayani, 2008) para incrementar el nivel de confianza de los modelos motivo de su investigación.

De esta manera fue que se calculó el nicho ecológico actual de la especie forestal $B$. coriacea cuya superficie es de $540.98 \mathrm{~km}^{2}$ como se muestra en la tabla 2, pero esta tenderá a disminuir en las proyecciones RCP8.5 del año 2050, con una pérdida de $319.04 \mathrm{~km}^{2}(-58.97 \%)$ y de $370.80 \mathrm{~km}^{2}(-68.54 \%)$ para el año 2070, como se observa en la figura 2. Pero, la pérdida más significativa se proyecta para el RCP6.0 del año 2050, con un $-86.19 \%$, lo que podría considerarse como una futura extinción regional de la especie por cambio climático (figura 3), lo que difiere de lo que Quesada et al., (2017) obtuvieron al modelar nichos ecológicos para cinco especies de plantas en zonas altas de Costa Rica (1500 a 3820 metros de altitud), en el que el valor más alto de pérdida de superficie de nicho ecológico en el escenario más pesimista (RCP8.5) sería de $-69 \%$. Está pérdida de nicho ecológico de $B$. coriacea podría deberse a la distribución altitudinal natural de la especie, entre los 3400 a 4500 metros (Reynel y Marcelo, 2009), ya que en los gradientes altitudinales el clima en combinación con otros factores ambientales ejercen influencia en la sobrevivencia de la flora a variaciones extremas de temperatura y precipitación (Guariguata y Matan, 2003). Esto permite explicar patrones de distribución de plantas (WoodWard, 1987) al registrar cambios en el tamaño y localización de las zonas de idoneidad ambiental para muchas especies en el mundo con cambios más severos en zonas montañosas, donde muchas especies tienden a elevar su límite altitudinal (Wilson et al., 2005).

Igualmente, se determinó un nicho ecológico actual de $1135.55 \mathrm{~km}^{2}$ para la especie $K$. lanceolata, especie forestal que Brako y Zarucchi (1993) describen su presencia en zonas con altitudes entre los 2000 y 4100 metros, mientras que Montesinos, et al., (2015) determinaron su distribución en el departamento de Arequipa entre los 2800 y 3040 metros de altitud. Es posible que este amplio rango altitudinal de desarrollo natural de la especie haga que se adapte rápidamente a diferentes condiciones ambientales y, por ello, su nicho ecológico tienda a ganar superficie a pesar de los cambios ambientales futuros, como se observa en la figura 3. De manera similar a la especie Ilex pallida en la investigación de Quesada et al. (2017), la cual tiene mayor distribución en el gradiente altitudinal de las cinco especies seleccionadas y que para los escenarios RCP 2.6 y RCP 4.5 el área aumenta con respecto al nicho ecológico actual, asegurando nuevos terrenos de zonas altas con características similares a las actuales. Esto hace suponer que la categoría de amenaza CR de $K$. lanceolata se deba principalmente al desarrollo de actividades antropogénicas como la agricultura, más que en los efectos del cambio climático. 
Buddleja coriacea

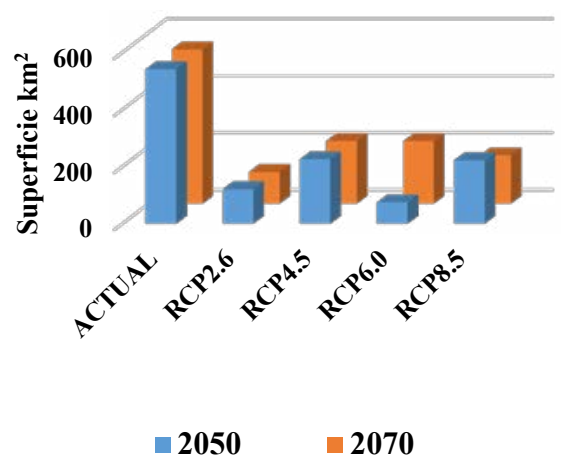

Haplorhus peruviana

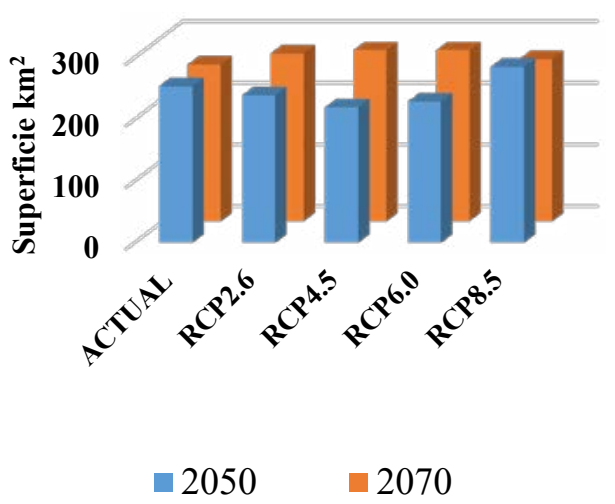

Carica candicans

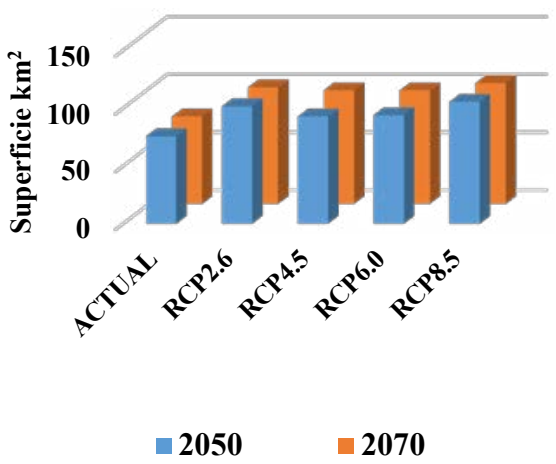

Kageneckia lanceolata

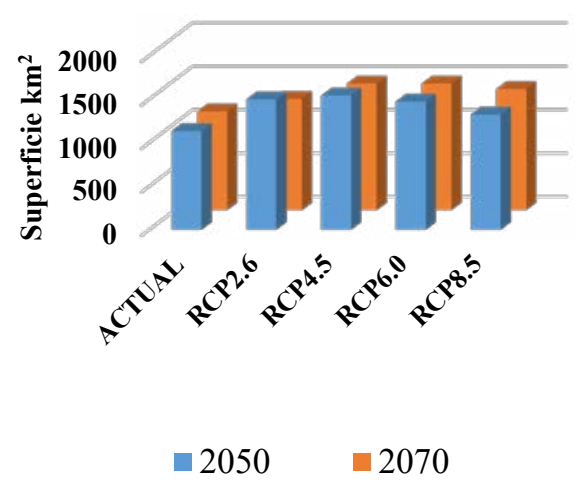

Weberbauerella brongniartioides

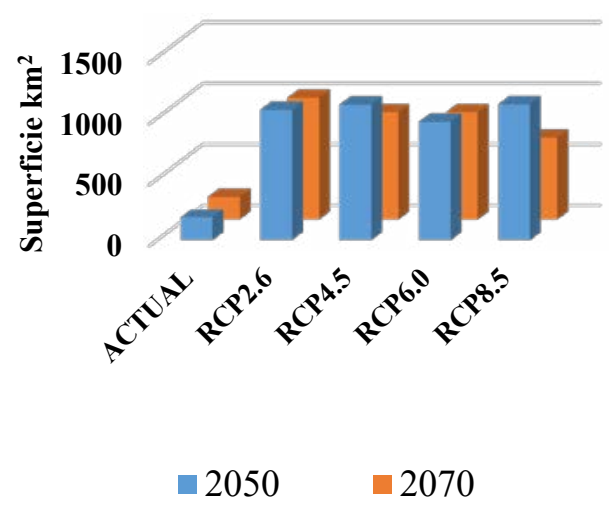

Figura 2. Tendencias en los cambios de la distribución actual de los nichos ecológicos de las especies categorizadas CR del departamento de Tacna en los escenarios RCP2.6, RCP4.5, RCP6.0 y RCP8.5 proyectados al 2050 y 2070 con MaxEnt. 

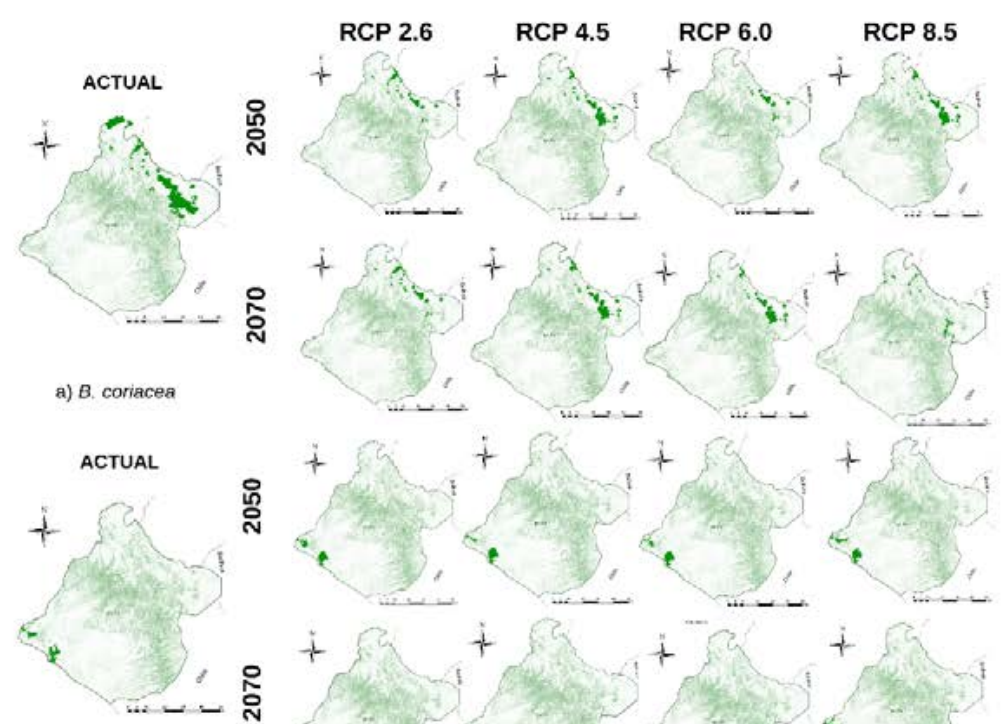

b) C. candicans
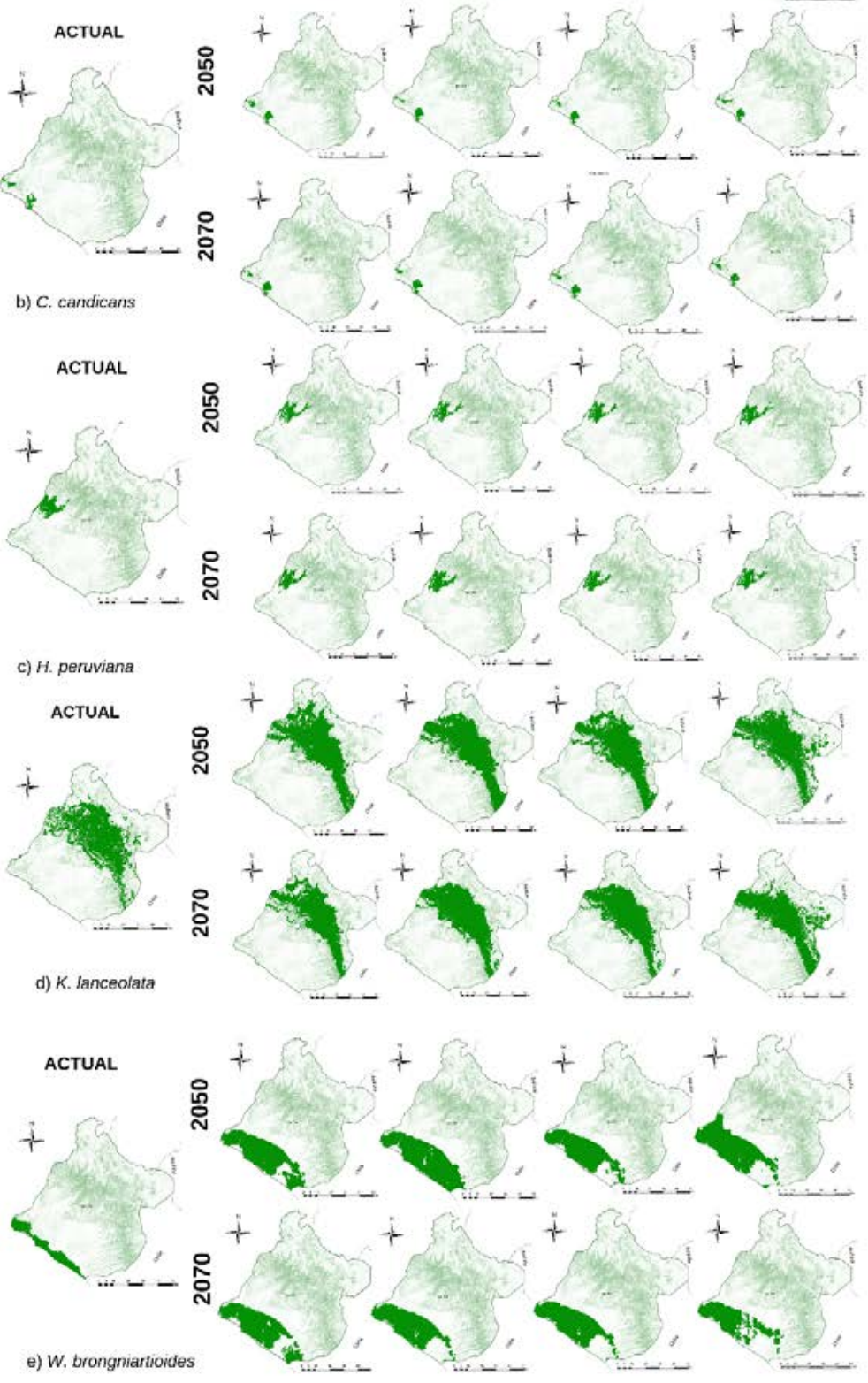

Figura 3. Distribución potencial de los nichos ecológicos actuales y proyectadas para los años 2050 y 2070, en cuatro escenarios de cambio climático: RCP2.6, RCP4.5, RCP6.0 y RCP8.5 de las especies a) B. coriacea b) C. candicans c) $H$. peruviana d) K. lanceolata e) W. brongniartioides. 
Con respecto a los nichos ecológicos obtenidos para las especies de flora silvestre categorizada que se distribuyen a menor altitud (de 0 a 2000 metros) que habitan zonas desérticas, costeras y de lomas, como C. candicans $\left(75.47 \mathrm{~km}^{2}\right), H$. peruviana $\left(254.05 \mathrm{~km}^{2}\right)$ y $W$. brongniartioides $\left(185.94 \mathrm{~km}^{2}\right)$, sus superficies tienden a mantenerse e incrementarse incluso en los RCP proyectados al año 2050 y 2070 como se muestra en la figura 3. En estas se observa que no se verán afectadas por las nuevas condiciones climáticas de los RCP futuros, lo que estaría asociada a sus ciclos vegetativos. Aunque Leiva, Galloso y Chang (2016) describieron a C. candicans como especie psicrófila (crece en lugares fríos), heliófila (requiere abundante luz del sol), higrófila (prefiere suelos húmedos, tierras negras con abundante humus), psamófila (puede desarrollarse en suelos arenosos, pedregosos) y argilícola (prospera en suelos arcillosos); aunque, por lo general, es una especie eutrofa que prefiere suelos con abundantes nutrientes. Esto también hace suponer que su categoría de amenaza CR se debe principalmente a la frecuente actividad antropogénica establecida en esta parte del país que el PNUD (2018) identifica como minería desregulada, invasiones de terrenos para viviendas, el tráfico de terrenos y el pastoreo no regulado de ganado.

Es así como al utilizar las mismas 19 variables bioclimáticas y otras de orientación, pendiente e índice topográfico de humedad y con la misma resolución espacial, pero para el área correspondiente a México, Estados Unidos y Centroamérica. Martínez et al. (2016) modelaron el nicho ecológico de las especies del género Abies (pinaceae) en México, obteniendo que las variables que más información aportaron en la mayor cantidad de especies fueron la Bio_19 (precipitación del cuarto más frío), común para seis especies, seguida de elevación, Bio_2 (intervalo promedio de temperaturas diurnas), Bio_18 (precipitación del cuarto más cálido) y pendiente, las cuales fueron compartidas por cinco especies. De igual manera, con las mismas variables bioclimáticas Quesada et al.
(2017) concluyeron que las variables más determinantes para el modelamiento de los nichos ecológicos de las especies utilizadas en su investigación son dependientes de las variables de temperatura, mientras que las de precipitación tienen aportes mínimos. En el caso del nicho ecológico actual de B. coriacea la variable predominante fue la Bio_1 (temperatura media anual) y las proyecciones de los RCP por la Bio_14 (precipitación del mes más seco) a excepción del RCP85 del año 2070, donde vuelve a influir la Bio_1. Para C. candicans todos los modelos obtenidos fueron influenciados por la Bio_12 (precipitación anual). Para $H$. peruviana la variable más influyente fue la Bio_7 (rango anual de temperatura), para K. lanceolata la Bio_18 (precipitación de cuarto mes más caluroso), al igual que para $W$. brongniartioides. Algunas otras variables de temperatura como Bio_9 (promedio en el trimestre más seco) obtuvieron valores altos solo para dos especies: $C$. candicans y $H$. peruviana.

Asimismo, en el Perú Osinfor (2016) realizó el modelamiento de la distribución potencial de nicho ecológico para 18 especies forestales en el departamento de Loreto, utilizando las mismas variables bioclimáticas y MaxEnt. En esta se concluye que la variable Bio_12 (precipitación total anual), es la que aporta mayor información a todos los modelos. Esta misma variable influyó principalmente en el modelo de C. candicans (tabla 2) y significa que para el ciclo vegetativo de estas especies es más importante el volumen anual acumulado de precipitación y no tanto las variaciones que por factores externos podrían alterar el régimen de lluvias en determinados meses del año.

Igualmente, durante el ciclo vegetativo de las cactáceas existe un predominio de la temperatura sobre la precipitación, por lo que se espera que en el modelamiento de sus nichos ecológicos estas variables influyan prioritariamente, como quedó evidenciado en el trabajo de Ibarra et al. (2016) en el que las variables bioclimáticas de temperatura fueron importantes en la distribución y persistencia de sus poblaciones y por sí solas fueron capaces 
de delimitar la distribución actual de Coryphantha chihuahuensis. Este mismo patrón sucedió en la especie forestal $H$. peruviana, cuyos nichos ecológicos derivaron exclusivamente de variables de temperatura, por lo que a pesar de no ser una cactácea, demuestra un similar ciclo vegetativo.

Con respecto a las variables de radiación solar, estas influyeron solo en el modelo actual del nicho ecológico de la especie desértica $W$. brongniartioides, pero no tuvieron mayor influencia en otras especies ni en los RCP proyectados. La variable ELE que representa a los metros de altitud del terreno tuvo valores medios de contribución en todos los modelos, pero sin ser predominantes.

Estos resultados permitieron conocer y analizar la distribución actual y potencial de los nichos ecológicos de las especies categorizadas CR de Tacna en los escenarios de cambio climático (IPCC, 2007), demostrándose que la altitud de distribución de las especies será influyente en la reducción del nicho ecológico frente a las variaciones de precipitación y temperatura proyectados para los RCP de los años 2050 y 2070, como se observa en la figura 3; mientras que habrá incremento del nicho ecológico de las especies de mayor rango altitudinal y que habitan zonas costeras. Sin embargo, considerando que los cambios de temperatura y precipitación en Tacna son muy variables en distancias muy cortas (Senamhi, 2010), existe una mayor probabilidad de error en las bases de datos de Worldclim en zonas de mayor altitud. Pero esto no significa que la información obtenida pueda ser útil para que las autoridades competentes encaren el cambio climático con mejores herramientas y se trabaje en lograr lo que Vegas (2010) señala en cuanto que las variaciones climáticas son una oportunidad para movilizar a la sociedad a enfrentar los problemas del subdesarrollo, capitalizar la economía, superar la pobreza y la desigualdad social en el ámbito local, regional y nacional, en especial con las especies nativas y endémicas.

En el departamento de Tacna Franco, Cáceres y Sulca (2019) determinaron 708 especies de flora vascular, siendo 92 endémicas. Los endemismos peruanos están principalmente vinculados a los Andes (Young, et al., 2002), destacando a B. coriacea como la especie más andina de la presente investigación. León, Pitman y Roque (2013) señalan que a través del conocimiento que se tenga sobre las especies endémicas de flora será posible discutir sobre el estado de conservación y el real futuro de la diversidad biológica, señalando que cuatro de las cinco especies categorizadas evaluadas en la presente investigación son endémicas (C. candicans, H. peruviana, B. coriacea y W. brongniartioides) mientras que se comparte la distribución de $K$. lanceolata con otros países de Sudamérica. Sin duda, el conocimiento generado sobre sus nichos ecológicos actuales y futuros de las especies endémicas evaluadas es un gran aporte para la conservación de la diversidad biológica. Por ello, se espera que a partir de los resultados obtenidos se empiece a discutir y promover el establecimiento estratégico de áreas naturales protegidas de administración pública o privadas en la superficie de los nichos ecológicos modelados, con fines de aprovechamiento, ecoturismo y conservación, como lo mencionan Ranganathan y Daily (2008), y con ella mejorar las oportunidades de desarrollo económico mediante el manejo de especies forestales o arbustivas en zonas de distribución silvestre o en plantaciones forestales. Estas pueden ser instaladas en zonas eriazas o agrícolas con las condiciones adecuadas a los nichos ecológicos de las especies evaluadas, a manera de recambio, como estrategia para la adaptación ante el cambio climático. Puesto que el IPCC (2013) señala que las tierras agrícolas serán afectadas a nivel productivo y, por ende, la seguridad alimentaria y el desarrollo económico deberán orientarse hacia la nueva estrategia denominada "Adaptación basada en ecosistemas" que propone la UICN en la publicación de Lhumeau y Cordero (2012).

Es por esto que las plantaciones forestales, en especial aquellas que trabajan con especies nativas, son una importante herramienta de mitigación y 
adaptación al cambio climático (Montesinos et al., 2019). De esta manera, es necesario lograr eficiencia en los proyectos de forestación y reforestación, por lo que es fundamental conocer las condiciones ambientales donde naturalmente habitan y que Mindreau y Zúñiga (2010) señalan como la exposición del terreno, humedad, altitud, pendiente, suelos, climas, flora acompañante y de conectividad; insumos que lograron obtenerse de las especies forestales evaluadas y que también pueden representar áreas potenciales para forestación.

El no haber considerado durante el modelamiento de nichos ecológicos variables de cambio de uso de suelo, es decir, el remplazo del nicho ecológico actual con infraestructura de la actividad antropogénica presente, ha hecho que el MaxEnt calcule superficies entre 254.05 a $1135.55 \mathrm{~km}^{2}$ (a excepción de $C$. candicans cuya superficie resultó en $75.47 \mathrm{~km}^{2}$ ), como se observa en la tabla 2 . Lo anterior difiere del concepto de la categoría de amenaza de la especie "En peligro crítico", que establece un límite de superficie de distribución

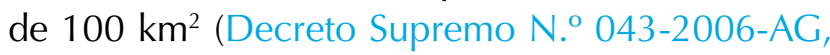
2006), lo que fue realizado por Kurpis, Serrato y Arroyo (2019), quienes usando un mapa de usos de la tierra concluyeron que las capacidades de dispersión de Tagetes lucida están mayormente amenazados por actividad humana.

Lo relevante de la presente investigación, y de los resultados obtenidos, es que se enmarcan en lo señalado por la Secretaría del Convenio de Diversidad Biológica (2004), la cual indica que las fuerzas motrices de los ecosistemas varían en el espacio y el tiempo, por lo que deben gestionarse y estudiarse a más de una escala. Esto evidencia la urgencia por analizar el territorio en los diferentes escenarios climáticos para predecir el futuro de las especies de flora y fauna que estarían en riesgo de extinción; como lo realizado por Ortíz, Restrepo y Páez (2014) al modelar la posible fluctuación de la distribución potencial de la tortuga Podocnemis lewyana Duméril en escenarios de cambio climático en Colombia Pliscoff y Fuentes (2011) al realizar una revisión de las nuevas herramientas y enfoques empleados al modelar la distribución de las especies y ecosistemas. Asimismo, se produce información científica de calidad sobre la distribución actual y futura de los nichos ecológicos de especies de flora con alto grado de amenaza y pocos antecedentes en investigación, que resulta pertinente y ayudará a las autoridades a planificar el territorio de acuerdo con los intereses globales de conservación, protección y manejo, orientado al desarrollo sostenible.

\section{CONCLUSIONES}

Los nichos ecológicos modelados para las cinco especies de flora silvestre categorizada del departamento Tacna tienen alto nivel de confianza. De igual manera, las variables ambientales más determinantes para cuatro de las cinco especies en la elaboración de sus modelos actuales y proyectados (2050 y 2070) fueron las que derivan de la precipitación, mientras que las variables de temperatura fueron altamente influyentes solo en el modelamiento del nicho ecológico de $H$. peruviana.

Es por ello que las políticas, actividades e iniciativas de conservación y ordenamiento territorial que se deriven de la información científica obtenida deberán ir orientadas a lograr resultados concretos en el escenario de mitigación RCP2.6 para garantizar el menor impacto del cambio climático a las especies. Siendo el nicho ecológico de $B$. coriacea el más variable en el tiempo, es necesario desarrollar programas de forestación y reforestación basadas en investigaciones científicas similares y aplicadas socioeconómicamente bajo la estrategia de adaptación basada en ecosistemas propuesta por la UICN mundialmente. Asimismo, para las especies evaluadas que habitan lomas costeras y desérticas, cuyo desarrollo futuro no se ve influenciada por las RCP, será necesario complementar este estudio con información de las actividades antropogénica que se desarrollan y que aproximarían aún más la distribución actual 
y proyectada de los nichos ecológicos con la finalidad de establecer mecanismos de ordenamiento territorial y de modalidades de conservación y reforestación.

Finalmente, la generación de escenarios como los calculados en el presente trabajo de investigación nos permite mejorar significativamente la comprensión de la dinámica de los nichos ecológicos de las especies de flora categorizada que habitan ecosistemas costeros, andinos y altoandinos a través del monitoreo de la biodiversidad, mostrándonos una alternativa más para la adecuada gestión y planificación territorial.

\section{CONTRIBUCIÓN POR AUTOR}

M.N. ideó la investigación; C.J. y J.I. sistematizaron y procesaron la información primaria y secundaria; M.N. elaboró los modelos de nichos ecológicos en MaxEnt y QGIS. Todos los autores realizaron el trabajo de campo, analizaron los datos, redactaron el manuscrito, contribuyeron a la discusión y comentaron los borradores.

\section{CONFLICTOS DE INTERESES}

Los autores declaran no tener conflicto de intereses.

\section{AGRADECIMIENTOS}

A la Universidad Latinoamericana Cima por el financiamiento del proyecto "Determinación de los cambios potenciales en la extensión y distribución geográfica de las zonas de vida de la región Tacna asociadas al cambio climático", aprobado mediante la resolución N. ${ }^{\circ}$ 020-2017-CU/ULC; así como al Herbario Takana y a la Gerencia Regional de Recursos Naturales y Gestión del Medio Ambiente del Gobierno Regional de Tacna por facilitar información de sus bases de datos.

\section{REFERENCIAS}

Brako, L. y Zarucchi, J. (1993). Catalogue of the Flowering Plants and gymnosperms of Peru. St. Louis, MO: Monographs in Systematic Botany, Vol. 45. St. Louis, MO: Missouri Botanical Garden. 1286 pp.

Castaño, C. (2002). Páramos y ecosistemas altoandinos de Colombia en condición hotspot y Global Climatic Tensor. Bogotá: Ideam. 387p.

Decreto Supremo N. ${ }^{\circ}$ 043-2006-AG (13 de julio del 2006). Aprueban categorización de especies amenazadas de flora silvestre. Diario Oficial El Peruano. Lima, Perú. Recuperado de

https://www.senace.gob.pe/wp-content/ uploads/2016/10/NAT-3-3-03-DS-043-2006-AG.pdf

Elith, J., Graham, C., Anderson, R., Dudík, M., Ferrier, S., Guisan, A., Hijmans, R., Huettmann, F., Leathwick, J. y Lehmann, A. (2006). Novel methods improve prediction of species distributions from occurrence data. Ecography, 29(2), 129-151.

https://doi.org/10.1111/j.2006.0906-7590.04596.x

Franco, J. (2013). Estudio poblacional de Carica candicans (papaya silvestre) en lomas de Morro Sama y lomas de Tacahuay. Gobierno Regional de Tacna. 56p. Recuperado de https://docplayer. es/65739526-Estudio-poblacional-de-carica-candicans-papaya-silvestre-en-lomas-de-morro-samay-lomas-de-tacahuay.html

Franco, J., Cáceres, C. y Sulca, L. (2019). Flora y vegetación del departamento de Tacna. Ciencia \& Desarrollo, 1(8), 23-30.

https://doi.org/10.33326/26176033.2004.8.143

Gobierno Regional de Tacna (2014). Plan de desarrollo regional concertado hacia el 2021. Tacna: Gobierno Regional de Tacna. 120p. Recuperado de

http://ww2.regiontacna.gob.pe/grt/documentos/2011/ personal/PDRC-2021.pdf

Guariguata, M. y Kattan, G. (2003). Ecología y conservación de bosques neotropicales. Cartago, Costa Rica: LUR. 691 p.

Hernández, B., Herrera, E., Cabrera., Delgado, A., Salazar, V., Bustamante, A., Campos, J. y Ramírez, J. (2016). Distribución potencial y características geográficas de poblaciones silvestres de Vanilla 
planifolia (Orchidaceae) en Oaxaca, México. Revista Biología Tropical, 64(1), 235-246.

\section{https://doi.org/10.15517/rbt.v64i1.17854}

Hijmans, R., Cameron, S., Parra, J., Jones, P. y Jarvis, A. (2005). Superficies climáticas interpoladas de muy alta resolución para áreas terrestres globales. Revista Internacional de Climatología, 25(15), 1965-1978.

https://doi.org/10.1002/joc.1276

Ibarra, I., Lebgue, T., Viramontes, O., Reyes, I., Ortega, J. y Morales, C. (2016). Modelo de nicho fundamental para Coryphantha chihuahuensis (cactaceae) en el estado de Chihuahua, México. Ecología Aplicada, 15(1), 11-17.

http://dx.doi.org/10.21704/rea.v15i1.578

IPCC (2007). Cambio climático 2007: informe de síntesis. En R. K. Pachauri y A. Reisinger (eds.), Contribución de los grupos de trabajo I, II y III al Cuarto Informe de evaluación del Grupo Intergubernamental de Expertos sobre el Cambio Climático. Ginebra, Suiza: IPCC. 104 p. Recuperado de

https://www.ipcc.ch/site/assets/uploads/2018/02/ ar4_syr_sp.pdf

IPCC (2013). Resumen para responsables de políticas. En: Stocker, T. F., D. Qin, G. K. Plattner, M. Tignor, S. K. Allen, J. Boschung, A. Nauels, Y. Xia, V. Bex y P.M. Midgley (eds.), Cambio climático 2013: bases físicas. Contribución del grupo de trabajo I al quinto Informe de Evaluación del Grupo Intergubernamental de Expertos sobre el Cambio Climático. Cambridge University Press, Cambridge, Reino Unido y Nueva York, EE. UU. Recuperado de

https://www.ipcc.ch/site/assets/uploads/2018/03/ WG1AR5_SPM_brochure_es.pdf

Kappelle, M. y Horn, S. (2005). Páramos de Costa Rica. Distribución, impacto humano y conservación de los páramos neotropicales. Santo Domingo de Heredia, Costa Rica: Instituto Nacional de Biodiversidad. 711 p. Recuperado de

https://www.academia.edu/27008250/P\%\%C3\%\%A1 ramos_de_Costa_Rica

Kurpis, J., Serrato, M. y Feria, T. (2019). Modeling the effects of climate change on the distribution of
Tagetes lucida Cav. (Asteraceae). Global Ecology and Conservation, 20, e00747.

https://doi.org/10.1016/j.gecco.2019.e00747

Leiva, S., Gayoso, G. y Chang, L. (2016). Carica candicans A. Gray (Caricaceae), una fruta utilizada en el Perú desde la época prehispánica. Arnaldoa, 23(2), 609-626.

https://doi.org/10.22497/arnaldoa.232.23212

León, B., Pitman, N. y Roque, J. (2013). Introducción a las plantas endémicas del Perú. Revista Peruana de Biología, 13(2), 9-22.

https://doi.org/10.15381/rpb.v13i2.1782

Lhumeau, A. y Cordero, D. (2012). Adaptación basada en ecosistemas: una respuesta al cambio climático. Quito: UICN. 21p. Recuperado de https:// portals.iucn.org/library/sites/library/files/documents/2012-004.pdf

Lobo, J., Jiménez, A. y Real, R. (2007). AUC: a misleading measure of the performance of predictive distribution models. Global Ecology and Biogeography, 17(2), 145-151.

https://doi.org/10.1111/j.1466-8238.2007.00358.x

Martínez, N., Aguirre, E., Eguiarte, L. y Jaramillo, J. (2016). Modelado de nicho ecológico de las especies del género Abies (pinaceae) en México: algunas implicaciones taxonómicas y para la conservación. Botanical Sciences, 94(1), 5-24.

https://doi.org/10.17129/botsci.508

Mendoza, A. (2006). Biodiversidad y sustentabilidad de las ciudades costeras: necesidad de mantener áreas naturales de vida silvestre en la ciudad. Biologist, 4, 4-5. Recuperado de

http://sisbib.unmsm.edu.pe/BVRevistas/biologist/v04_ n1/vol4n1_2006.pdf

Minam (2015). Estrategia nacional ante el cambio climático. Lima: Ministerio del Ambiente. 88 p.

Minam (2018). Listado de especies de flora silvestre Cites-Perú. Lima: Ministerio del Ambiente. 236 p. Recuperado de

http://www.minam.gob.pe/simposio-peruano-de-especies-cites/wp-content/uploads/sites/157/2018/08/ Listado-FAUNA-CITES-FINAL.pdf

Mindreau, M. y Zúñiga, C. (2010). Manual de forestería comunitaria de alta montaña - experiencias 
de reforestación con Polylepis sp en el corredor de Conchucos-Ancash. Huaraz: Instituto de Alta montaña. $30 \mathrm{p}$.

Montesinos, D., Sýkora, K., Quipuscoa, V. y Cleef, A. (2015). Species composition and phytosociology of xerophytic plant communities after extreme rainfall in South Peru. Phytocoenologia, 45(3), 203-250.

https://doi.org/10.1127/phyto/2015/0023

Montesinos, D., Núñez del Prado, H., Bustamante, T., Brian, J., Álvarez, E., Borgoño, A. y Riveros, G. (2019). Diversidad florística, comunidades vegetales y propuestas de conservación del monte ribereño en el río Chili (Arequipa, Perú). Arnaldoa, 26(1), 97-130.

https://doi.org/10.22497/arnaldoa.261.26106

Morales, N. (2012). Modelos de distribución de especies: Software MaxEnt y sus aplicaciones en Conservación. Revista Conservación Ambiental, 2(1), 1-3. Recuperado de https://issuu.com/fundacionecomabi/ docs/revista_conservaci_n_ambiental_mas

Nasa (2019). Imagery by Jesse Allen, Nasa's Earth Observatory, using data from the General Bathymetric Chart of the Oceans (GEBCO) produced by the British Oceanographic Data Centre. Recuperado de: https://visibleearth.nasa.gov/view.php?id=73934

Narayani B. (2008). Tool for Partial-ROC (Biodiversity Institute, Lawrence, KS), ver 1.0.

Ordenanza Regional N..$^{\circ}$ 005-2015-CR/GOB. R.REG. T.TACNA (14 de enero del 2015). Aprueban el instrumento de Gestión Ambiental Regional denominado: "Estrategia Regional de Cambio Climático (ERCC) de Tacna" y dictan otras disposiciones. Diario Oficial El Peruano. Lima, Perú. Recuperado de

https://busquedas.elperuano.pe/normaslegales/aprueban-el-instrumento-de-gestion-ambiental-regional-denomi-ordenanza-no-005-2015-crgobregtacna-1273301-1/

Ordenanza Regional N. ${ }^{\circ}$ 016-2012-CR/GOB. R.REG. T.TACNA (18 de enero del 2013). Aprueban la zonificación ecológica y económica de Tacna. Diario Oficial El Peruano. Lima, Perú. Recuperado de https://busquedas.elperuano.pe/ normaslegales/modifican-la-ordenanza-re- gional-n-016-2012-crgobregtacna-ordenanza-n-018-2013-crgobregtacna-1053975-1/

Ortega, M. y Townsend, A. (2008). Modeling ecological niches and predicting geographic distributions: a test of six presence-only methods. Revista Mexicana de Biodiversidad, 79(1), 205-216.

http://dx.doi.org/10.22201/ib.20078706e. 2008.001.522

Ortíz, C., Restrepo, A. y Páez, V. (2014). Distribución potencial de Podocnemis lewyana (Reptilia: Podocnemididae) y su posible fluctuación bajo escenarios de cambio climático global. Acta Biológica Colombiana, 19(3), 471-481.

https://doi.org/10.15446/abc.v19n3.40909

Osinfor (2013). Modelamiento espacial de nichos ecológicos para la evaluación de presencia de especies forestales maderables en la amazonía peruana. Lima: Osinfor. 82 p. Recuperado de

https://www.osinfor.gob.pe/portal/data/destacado/adjunto/modelamiento_nichos_ecologicos.pdf

Osinfor (2016). Modelamiento de la distribución potencial de 18 especies forestales en el departamento de Loreto. Lima: Osinfor. 90 p. Recuperado de

https://www.osinfor.gob.pe/wp-content/ uploads/2016/06/modelamiento-de-18-especies-21-JUNIO.pdf

Peterson, A., Papes, M. y Soberón, J. (2008). Rethinking receiver operating characteristic analysis applications in ecological niche modeling. Ecological Modelling, 213(1), 63-72.

https://doi.org/10.1016/j.ecolmodel.2007.11.008

Phillips, S., Anderson, R. y Sphapire, R. (2006). Maximum entropy modeling of species geographic distributions. Ecological Modelling, 190(3), 231-259.

https://doi.org/10.1016/j.ecolmodel.2005.03.026

Phillips, S. y Dudík, M. (2008). Modeling of species distribucions with MaxEnt: new extensions and a comprehensive evaluation. Ecography, 31(2), 161-175.

https://doi.org/10.1111/j.0906-7590.2008.5203.x

Pliscoff, P. y Fuentes, T. (2011). Modelación de la distribución de especies y ecosistemas en el tiempo y en el espacio: una revisión de las nuevas herramientas y enfoques disponibles. Revista de Geografía Norte Grande, 48(1), 67-79. 
PNUD (2018). Retos y oportunidades en la conservación de las lomas de Lima metropolitana. Lima: PNUD. 20 p. Recuperado de

http://www.pe.undp.org/content/peru/es/home/library/ eba-lomas.html

Pumasupa, M. (2018). Cuantificación de la captura de carbono de la especie forestal Haplorhus peruviana Carzo como servicio ambiental en el Valle de Cinto, provincia Jorge Basadre, región Tacna, Perú (trabajo de grado, Ingeniería. Ambiental). Universidad Privada de Tacna, Perú. 132 p. Recuperado de

http://repositorio.upt.edu.pe/handle/UPT/558

QGIS Development Team (2019). QGIS Geographic Information System. Open Source Geospatial Foundation Project. Recuperado de

https://qgis.org

Quesada, M., Acosta, L., Arias, D. y Rodríguez, A. (2017). Modelación de nichos ecológicos basado en tres escenarios de cambio climático para cinco especies de plantas en zonas altas de Costa Rica. Revista Forestal Mesoamericana Kurú, 14(34), 1-12. https://doi.org/10.18845/rfmk.v14i34.2991

Ranganathan, J. y Daily, G. (2008). La biogeografía del paisaje rural: oportunidades de conservación para paisajes de Mesoamérica manejados por humanos. En: C. A. Harvey y J. C. Sáenz (eds.), Evaluación y conservación de biodiversidad en paisajes fragmentados de Mesoamérica (pp. 15-30). Santo Domingo de Heredia, Costa Rica: Instituto Nacional de Biodiversidad.

Reynel, C. y Marcelo, J. (2009). Árboles de los ecosistemas forestales andinos. Manual de identificación de especies. Lima: Serie Investigación y Sistematización N. ${ }^{\circ}$ 9. Programa Regional Ecobona-Intercooperation. 163 p. Recuperado de

http://www.asocam.org/sites/default/files/publicaciones/files/02190ca87f921d9feb250ae672ae6653.pdf

Reynel, C. y León, J. (1990). Árboles y arbustos andinos para agroforestería y conservación de suelos. Lima: Proyecto FAO, Holanda, DGFF. 361 p.

Reynoso, R., Pérez, M., López, W., Hernández, J., Muñoz, H., Cob, J. y Reynoso, M. (2018). El nicho ecológico como herramienta para predecir áreas potenciales de dos especies de pino. Revista Mexicana de Ciencias Forestales, 9(48), 47-68.

https://doi.org/https://doi.org/10.29298/rmcf.v8i48.114

Sagástegui, A., Rodríguez, E. y Arroyo, S. (2007). Plantas promisorias: el mito o papaya silvestre. Innova Norte, 1(1), 109-119. Recuperado de

https://www.academia.edu/36380903/Plantas_ Promisorias_El_mito_o_Papaya_Silvestre

Secretaría del Convenio sobre la Diversidad Biológica (2004). Enfoque por ecosistemas., Montreal: Convenio sobre Diversidad Biológica. 50 p. Recuperado de

https://www.cbd.int/doc/publications/ea-text-es.pdf

Senamhi (2010). Resumen técnico del cambio climático nacional. Lima: Senamhi. 23 p. Recuperado de https://www.senamhi.gob.pe/

Shcheglovitova, M. y Anderson, R. (2013). Estimating optimal complexity for ecological niche models: A jackknife approach for species with small simple sizes. Ecological Modelling, 269, 9-17.

https://doi.org/10.1016/j.ecolmodel.2013.08.011

UICN (2012). Categorías y criterios de la lista roja de la UICN. Versión 3.1. Segunda edición. Gland, Suiza y Cambridge, Reino Unido: UICN. 34 pp. Recuperado de

https://portals.iucn.org/library/sites/library/files/documents/RL-2001-001-2nd-Es.pdf

Vegas, I. (2010). Cambio climático en el Perú. Regiones del sur del Perú. Lima, Perú: Fundación Manuel J. Bustamante de la Fuente. 126 p. Recuperado de

http://www. fundacionmjbustamante.com/wp-content/uploads/2012/06/Cambio-Climatico-Regiones-del-Sur.pdf

Wan, J., Wang, C., Yu, J., Nie, S., Han, S., Liu, J., Zu, Y. y Wang, Q. (2015). Developing conservation strategies for Pinus koraiensis and Eleutherococcus senticosus by using model-based geographic distributions. Journal of Forestry Research, 27(2), 389-400.

https://doi.org/10.1007/s11676-015-0170-5

Weberbauer, A. (1945). El mundo vegetal de los Andes peruanos. Lima: Ministerio de Agricultura. 776 pp.

Wilson R., Gutiérrez D., Gutiérrez J., Martínez D., Agudo R. y Monserrat, V. (2005). Changes to the 
elevational limits and extent of species ranges associated with climate change. Ecological Letters, 8(11), 1138-1146.

https://doi.org/10.1111/j.1461-0248.2005.00824.x

Woodward, F. (1987). Stomatal Numbers Are Sensitive to Increases in $\mathrm{CO} 2$ from Pre-Industrial Levels. $\mathrm{Na}$ ture, 327, 617-618.

http://dx.doi.org/10.1038/327617a0

Worldclim (2016). Free climate data for ecological modeling and GIS. Worldclim-Global Climate Data. Recuperado de

http://www.worldclim.org/
World Bank Group (2019). Solar resource data obtained from the Global Solar Atlas, owned by the World Bank Group and provided by Solargis. Recuperado de

https://globalsolaratlas.info/

Young, K., Ulloa, C., Luteyn, J. y Knapp, S. (2002). Plant evolution and endemism in Andean South America: An Introduction. Bot. Rev., 68(1), 4-21.

https://doi.org/10.1663/0006-8101(2002)068[0004:peaeia]2.0.co;2

\section{(c) (i) (?)

